\title{
Correspondence
}

\section{Mechanism for the synergistic effect of rapamycin and resveratrol on hyperinsulinemia may involve the activation of protein kinase $B$}

\author{
$\mathrm{J} \mathrm{Chen}^{\star, 1}$ and X-F Huang ${ }^{2}$
}

Cell Death and Disease (2013) 4, e680; doi:10.1038/cddis.2013.196; published online 20 June 2013

Subject Category: Neuroscience

\section{Dear Editor,}

We read with great interest Leontiev et al.'s paper entitled 'Resveratrol potentiates rapamycin to prevent hyperinsulinemia and obesity in male mice on high-fat diet' recently published in Cell Death \& Disease. ${ }^{1}$ Their finding that rapamycin and resveratrol have a synergistic effect is important for treating insulin resistance. They showed that rapamycin inhibited mammalian target of rapamycin (mTOR) activity, while resveratrol inhibited S6 kinase (S6K). We think that these compounds may exert their effects through activating protein kinase B (Akt), a key regulator of insulin sensitivity. Both the inhibition of mTOR by rapamycin and of S6K by resveratrol could result in the activation of Akt due to the release of feedback inhibition on the insulin receptor substrates (IRS), which are stimulators of Akt.

Insulin resistance is a pathophysiological condition often occurring in diabetes, metabolic syndrome and obesity. As there is a high incidence of diabetes and obesity, there is also insulin resistance in a large proportion of the population. In insulin resistance, adipocytes and muscle cells are no longer sensitive to insulin and thus their uptake of glucose is reduced. ${ }^{2}$ Hepatic cells also fail to convert glucose into glycogen. As a result, blood glucose levels are increased, and insulin secretion is correspondingly increased, causing hyperinsulemia. It has been recognized that hyperinsulinemia is associated with many co-morbidities such as cancer and heart disease. Insulin can activate several intracellular signal pathways-including phosphoinositide 3-kinase (PI3K)/Akt and mitogen-activated protein kinase (MAPK)-to increase cell proliferation and decrease apoptosis, thus facilitating carcinogenesis and leading to poorer prognosis to cancer therapy. ${ }^{3}$ Therefore, it is important to control hyperinsulinemia.

Akt has been demonstrated to be a key regulator in the insulin-induced transportation of glucose into cells. The downstream target protein AS160 can increase the translocation of glucose transporter 4 (Glut 4) from intracellular vesicles to the plasma membrane. ${ }^{4}$ In mice, an Akt knockdown is sufficient to cause a diabetic phenotype. ${ }^{5}$ In humans, the loss of Akt function due to a mutation is associated with diabetes. ${ }^{6}$ In Leontiev et al.'s paper, it is shown that rapamycin and resveratrol have a synergistic effect in lowering hyperinsulinemia. ${ }^{1}$ The authors explored the effect of rapamycin and resveratrol on two components of the PI3K/Akt pathway, mTOR and S6K. However, their effects on the levels of phosphorylated Akt (pAkt) were not examined. We think that rapamycin and resveratrol may synergistically activate Akt to increase insulin sensitivity in mice fed with a high-fat diet.

The insulin/PI3K/Akt signaling is negatively regulated by a feedback loop acting on $\mathrm{Akt}^{3}$ In this pathway, insulin activates the insulin receptor that phosphorylates IRS, leading to the activation of the PI3K/Akt pathway. One of the Akt target proteins is mTOR, which regulates S6K. The S6K negatively regulates IRS, leading to the inhibition of the Akt activity. ${ }^{3}$ In Leontieva et al.'s study, rapamycin was shown to inhibit mTOR, which should lead to decreased phosphorylated S6K (pS6K), and subsequently result in the activation of Akt (Supplementary Figure 1).

Resveratrol can inhibit pS6K directly as shown in Leontieva et al.'s study. It could also cause the activation of Akt (Supplementary Figure 1), which was not examined in the study. Nevertheless, another study has shown that resveratrol does increase pAkt in muscle cells in type 2 diabetes. ${ }^{7}$ Inhibition of AMP-activated protein kinase by resveratrol in muscle cells may also contribute to an increase in pAkt. $^{8}$ The combinatorial application of rapamycin and resveratrol may result in a stronger inhibition of pS6K, and thus a higher activation of pAkt. This may explain the synergistic effect of the two compounds used in this study.

\section{Conflict of Interest}

The authors declare no conflict of interest.

1. Leontieva OV et al. Cell Death \& Disease 2013; 4: e472

2. Yki-Jarvinen $\mathrm{H}$ et al. Diabetes 1990; 39: 157-167.

${ }^{1}$ School of Biomedical Sciences, University of Queensland, St. Lucia, Queensland, Australia and ${ }^{2}$ lllawarra Health and Medical Research Institute, University of Wollongong, Wollongong, Australia

*Corresponding author: J Chen, School of Biomedical Sciences, University of Queensland, Sir William MacGregor Building, St. Lucia, Queensland 4072, Australia. Tel: +61 7 33651409; Fax: +61 7 33652398; E-mail: j.chen4 @uq.edu.au 
3. Chen J. Obesity Rev 2011; 12: 1063-1070.

4. Sano H et al. J Biol Chem 2003; 278: 14599-14602.

5. Mauvais-Jarvis F, Kulkarni RN, Kahn CR. Clin Endocrinol 2002; 57: 1-9.

6. George S et al. Science 2004; 304: 1325-1328.

7. Brasnyo $\mathrm{P}$ et al. Br J Nutr 2011; 106: 383-389.

8. Skrobuk P et al. Diabetologia 2012; 55: 3051-3060.
Cell Death and Disease is an open-access journal published by Nature Publishing Group. This work is licensed under a Creative Commons Attribution-NonCommercialNoDerivs 3.0 Unported License. To view a copy of this license, visit http://creativecommons.org/licenses/by-nc-nd/3.0/

Supplementary Information accompanies this paper on Cell Death and Disease website (http://www.nature.com/cddis) 